\title{
Innovation of Traditional Creation Wisdom in Intelligent Age
}

\author{
Zonghua $\mathrm{Zhu}^{1}$ \\ ${ }^{1}$ Jingchu University of Technology, Jingmen, Hubei, 448001, China
}

\begin{abstract}
In recent years, with the idea of carrying forward traditional culture gradually gaining popularity, people pay more attention to the traditional creation wisdom contained in traditional artifacts, and on this basis, actively explore more innovative design methods, so as to realize the organic combination of traditional creation wisdom and intelligent era, so that traditional creation wisdom can be better inherited and carried forward. Based on this, this paper discusses the traditional creation wisdom, combines the development and improvement of modern times, takes the design of artistic furniture as a case, the paper analyzes how to combine and innovate the traditional creation wisdom in the product design under the current intelligent era by the combination of traditional creation wisdom and the emerging "Unity 3D" technology, in order to better inherit and carry forward the traditional creation wisdom.
\end{abstract}

\section{Introduction}

The traditional creation wisdom is an important part of China's excellent traditional culture, which contains profound ideological essence and value essence. These ideological values still have a certain degree of advancement in modern society, and they must also have important application value in modern society, so we still need to make further in-depth research on it. Of course, because the background of these creative wisdom has changed significantly, in order to better promote its application in contemporary life and make traditional creative wisdom deeply integrate with modern society, we need to think about how to innovate traditional creative wisdom based on the background of intelligent era, and truly realize "making the past serve the present", so that the wisdom contained in traditional creative wisdom can really inspire modern innovative design, promote China's leading role in the design field, and then make our traditional creative wisdom..

\section{Overview of the Traditional Creations Wisdom}

\subsection{Connotation of Traditional Creation Wisdom}

The connotation of traditional creation wisdom is divided into two levels: surface and deep. From the surface dimension, the generation of traditional creation wisdom can be said to be a historical necessity, which is based on people's basic living needs[1]. In the development of history, the wisdom of the working people has created a large number of traditional instruments, which generally have high practical value and play a great role in promoting productive labor. Behind the scenes, each instrument has its own unique background story, which records the birth and development of civilization[2].

With the gradual development of traditional culture, traditional creation wisdom has gradually broken through the category of original function and gradually developed towards the dimension of deep connotation. It not only focuses on basic practical value, but also usually explains the layout structure of objects with unique creativity and pursues natural design goals, which contains more historical culture behind it. Therefore, it is necessary to carefully study the traditional creation wisdom, with the emphasis on tracing back to the source, making an indepth study on it from the level of history and culture, understanding the value of these traditional creation wisdom in essence, and comparing it with modern design to realize the inheritance and innovation of traditional wisdom of creation [3].

\subsection{Characteristics of the Traditional Creation Wisdom}

2.2.1 Make the Best Use of Everything. Making the best use of everything can be said to be the primary feature of the traditional creation wisdom. Since the origin of traditional creation, this feature has run through the whole process of traditional creation. It is mainly based on the following two reasons: first, the low level of productivity in ancient times; second, people summed up a lot of natural laws in their daily production and life. The characteristics of making the best use of everything were reflected in ancient China. In some agricultural civilization sites in China, archaeologists discovered the accumulation layer and carbonization layer of rice husk, which proved that rice husk, a by-product of rice threshing, had been fully utilized at that time, and there was no waste 
of materials. It can be seen that the ancients have made full use of all kinds of materials donated by nature in the unfavorable environment with extremely limited conditions, and minimized the waste of materials, which is quite similar to the concept of "zero waste" in modern society. Obviously, this traditional creation wisdom is also of great value to modern society [4].

2.2.2 Function First. In the traditional concept of creation, there are not too many requirements for form and structure, which are usually more concise, but more emphasis on the actual function of objects. For a long time, the traditional way of creation in our country has been influenced by this concept, and has continued to this day, becoming a design concept which is obvious and excellent in the wisdom of traditional creation and worth inheriting[5].

2.2.3 Tibetan Ritual. Since ancient times, China has enjoyed the reputation of "an ancient civilization" and "a country of etiquette", which is also related to many etiquette systems in ancient China. China's traditional etiquette system is also based on various traditional etiquette objects. Such as bronzes, sacrificial utensils and court display utensils in Shang and Zhou Dynasties. Generally speaking, ceremonial objects are generally characterized by majesty and solemnity. These ceremonial objects have been given more meaning of "ceremony" in the development of etiquette system. With the development of the times, the cultural value behind these ceremonial objects has been further sublimated, reflecting people's morality and ideals, as well as the rules and order of the real world.

\section{The Innovative Strategy of Chinese Traditional Creation Wisdom in the Intelligent Era_-Taking Art Furniture Design as an Example}

In recent years, with the further development of the Internet, people have entered an intelligent era, and artificial intelligence technology has been able to assist human decision-making. Obviously, the future era will develop from the traditional ternary space to the quaternary space composed of human, physical world, intelligent machine and virtual information world. Under this development background, the future development of the times will pay more attention to meeting people's individual needs. Although most of the traditional artifacts have entered the museum, the traditional wisdom of creation should never disappear, but should be combined with various new technologies, so that our traditional wisdom of creation can exert greater influence in this intelligent era and promote the innovation of design and creation in our country. It is predicted that with the further development of science and technology, the design of objects in the future will be carried out under the framework of the Internet of Things system[6]. During the process of using products, users will have more frequent data and information interaction with products, and then through the integration and analysis of big data technology, more effective suggestions will be provided for users, which will improve the product use efficiency and further improve the user experience. At present, in daily life, there are still some shortcomings in many product designs, which need to be solved with more creative ideas. For this reason, traditional wisdom of creation can be used to solve them [7-8].

\subsection{Overall design position}

Based on the current market research results and project demand analysis, the design has the following positioning: First, positioning the product category, which mainly aims at the living room furniture in art furniture, such as sofa and coffee table, because it is frequently used furniture. Second, the overall positioning, which mainly uses the virtual design method to determine the material, shape and texture individually. Thirdly, we should make a functional orientation to ensure that the traditional wisdom of creation contained in it can adapt to the changes of modern lifestyle and meet the requirements of ergonomics, so as to get final recognition. Fourth, the structural orientation, in terms of structure, the traditional mortise and tenon structure in the traditional wisdom of creation is still used to realize the connection of furniture.

\subsection{Overall development process}

This design mainly uses the emerging "Unity 3D" virtual reality technology in computer technology to design and display the target products, and will use the rendering technology of Unity 3D platform to improve the simulation effect of art furniture virtual design. This is helpful to meet the individual needs of different groups for furniture design, and it also provides new ideas and methods for subsequent design work[7].

\subsubsection{Establish engineering and import resources.} First, start Unity 3D, create a new engineering project, and then click the Asset packages... button, and the Unity resource package required by the new project will be automatically introduced. Finally, click the Create Project button to create a new Unity 3D project, and import a specific Unity 3D resource package to improve work efficiency. Of course, it should be noted that the path and name of the project must use English instead of Chinese, otherwise mistakes will easily occur.

3.2.2 Scene construction and optimization. After the Unity $3 \mathrm{D}$ resources are imported, you can directly see the imported resources such as 3D models, mapping materials and texture pictures in the Assets directory of Project view, and then click the File-New Scene option to create a new scene. In the process of scene construction and optimization, in order to ensure a more realistic simulation effect, the maps and materials of art furniture should be baked first. After baking, the model can be directly dragged into the newly created scene view. 
In the view, three different scenes need to be established, each scene corresponds to a different style of art furniture, and the three styles can be switched between each other. After adjusting the positions of the three different models, you can add lights and adjust the cameras in the scene. Because each scene corresponds to different types of content, it is necessary to flexibly adjust the light position, size and camera angle before setting up the scene. When the three styles can be switched freely, the material can be selected and converted. This function is mainly realized by $\mathrm{C \#}$ script, and the key segment code is as follows.

//Color wheels

$\mathrm{m}$ ColorWheels=new ColorWheelsSettings

\{

mode $=$ FindSetting $($ Settings $\mathrm{x})=>\mathrm{x}$.colorWheels.mode $)$

$\log =$ FindSetting $($ Settings $\mathrm{x})=>\mathrm{x}$.colorWheels.log $)$

linear $=$ FindSetting $(($ Settings $\mathrm{x})=>\mathrm{x}$.colorWheels.linear $)$

//Curves

m_Curves $=$ new CurvesSettings

master $=$ FindSetting $($ Settings $\mathrm{x}) \Rightarrow$ x.curves.master.curve $)$,

red $=$ FindSetting $(($ Settings $\quad \mathrm{x}) \Rightarrow$ x.curves.redxurve $)$,

green $=$ FindSetting $(($ Settings $\mathrm{x}) \Rightarrow$ x.curves.green.curve $)$,

blue $=$ FindSetting $($ (Settings $\mathrm{x}) \Rightarrow$ x.curves.blue.curve $)$,

hueVShue $=$ FindSetting $($ (Settings $\mathrm{x}) \Rightarrow \mathrm{x}$.curves.hueVShue.curve $)$,

hueVSsat $=$ FindSetting $(($ Settings $\mathrm{x}) \Rightarrow$ x.curves.hueVSsat.curve $)$,

satVSsat $=$ FindSetting $(($ Settings $\mathrm{x}) \Rightarrow$ x.curves.satVSsat.curve $)$,

lumVSsat $=$ FindSetting $($ (Settings $\mathrm{x}) \Rightarrow$ x.curves.lumVSsat.curve $)$,

currentEditingCurve $=$ FindSetting $($ Settings $\mathrm{x}) \Rightarrow$ x.curves.e

CurrentEditin gCurve)

curveY $=$ FindSetting $(($ Settings $\quad x) \Rightarrow$ x.curves.e_CurveY $)$ ?

curveR $=$ FindSetting $($ (Settings $x) \Rightarrow$ x.curves.e - CurveR $)$,

curveG $=$ FindSetting $($ Settings $\mathrm{x}) \Rightarrow \mathrm{x}$. cnrves.e CurveG),

curveB $=$ FindSetting $($ Settings $\mathrm{x}) \Rightarrow$ x.curves.e CurveB $)$ ;

Finally, the "first person character controller" is directly imported into the environment, and the user can adjust the controller parameters according to the needs of human body size. After all the above steps are completed, the game view can be run to roam the virtual environment, and users can intuitively see the form and material of furniture virtual design and its matching degree with the environment in the virtual environment[8].

\subsubsection{Visual design and implementation of the} interface. In Unity 3D, most interactive design processes will be carried out. Obviously, in terms of visual design and implementation of interface, it mainly includes several modules: the choice of artistic furniture style, the choice of artistic furniture material, the display of furniture details and indoor roaming.

In the interface design, it is mainly embodied in the form of icons and information pop-ups. The entry interface of the system should contain some summary information about furniture virtual design; and in the main interface of the system (that is, the first interface after entering the system), the detailed display contents should be included.

\subsubsection{Publishing and display of virtual design. After} the virtual design of art furniture is completed, it needs to be released and displayed on the PC platform. The work in this link is relatively simple. First, click the "PC" icon in "Build Setting" under the menu bar, then set "icon" in "Player Setting", click "Build", export the relevant files in the format of "exe", rename them, save them in a specific folder, and then run them directly on the PC and realize the corresponding functions.

\section{Conclusion}

On the whole, Chinese traditional creation wisdom is profound, and there are still many outstanding points which have not been discovered by us. These outstanding points undoubtedly still have important practical significance for the innovation of modern product design. Looking at the current product design, it is clear that it has further developed in the direction of humanistic connotation. Therefore, it is still a problem worth studying how to make the traditional wisdom of creation with more humanistic connotation manifest on more artifacts from the technical level. Of course, there are more than one standard answer to things. The current excellent design cases should play the role of attracting valuable suggestions. In the future, further research and innovation in related fields are needed to better promote the development and inheritance of traditional creation wisdom.

\section{Acknowledgments}

Fund-The Second Batch of Scientific Research Teams of Jingchu University of Technology-Research on Traditional Creation Culture in Central Hubei (No. TD202005).

\section{References}

1. Wu Xinlin. The Form of Chinese Traditional Creation Wisdom and Its Modern Translation[A]. Tongji University, Hunan University of Science and Technology, National Major Social Science Projects Research on Chinese Artisan Cultural System and Its Inheritance and Innovation Research Group.Symposium on Chinese Design Theory and Technological Innovation_—Proceedings of the 4th Chinese Design Theory and the 4th National Highend Forum on Cultivating Chinese Artisans[C].Tongji University, Hunan University of Science and Technology, National Major Social Science Projects Research on Chinese Artisan Cultural System and Its Inheritance and Innovation Research Group: College of Design and Innovation, Tongji University, 2020:8.

2. He Sheng.Study on Enlightenment and Innovative Design of Chinese Traditional Creation Wisdom in the Intelligent Age[J].Packaging Engineering,2020,41(16):353-359.

3. Li Bing, Yan Siyuan.The Path Study of Traditional Creation Wisdom Enlighten Modern Product Innovation Engineering,2020,41(22):95-99.

4. Peng Wei. Research on the Application of Traditional Creation Wisdom in Daily Home Product Design[D].Sichuan Fine Arts Institute,2019. 
5. Liang Bing.Exploration on Creation Wisdom and Design Thought of Traditional Handicrafts[J].Art Panorama,2017(11):70-71.

6. Wu Xinlin.From The Artificers' Record to T'ien-kung k'ai-wu:The Concession of Chinese Traditional Creation Thought from the Perspective of Art Comparison[J].Art \& Design Research,2018(03):7983.

7. Pan Tianbo.Chinese Examination of Technology: History, Logic and Form[J].Ethnic Art Studies,2019,32(04):91-98.

8. Yu Ding.Chinese School of Creation of Art Management in 5G Times[N].Chinese Culture News,2019-08-18(006). 